
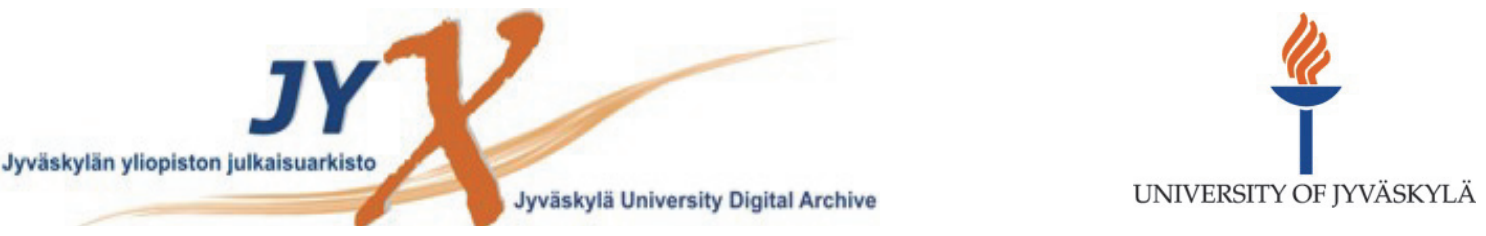

This is an electronic reprint of the original article. This reprint may differ from the original in pagination and typographic detail.

\author{
Author(s): Ertoprak, A.; Cederwall, B.; Jakobsson, U.; Nyako, B. M.; Nyberg, J.; Davies, P.; Doncel, \\ M.; De France, G.; Kuti, I.; Napoli, D. R.; Wadsworth, R.; Chugre, S. S.; Raut, R.; Akkus, \\ B.; Al-Azri, H.; Algora, A.; De Angelis, G.; Ata, A.; Bäck, T.; Boso, A.; Clement, E.; \\ Debenham, D. M.; Dombradi, Zs.; Erturk, S.; Gadea, A.; Moradi, F. Ghazi; Gottardo, A.; \\ Huyuk, T.; Ideguchi, E.; Jaworski, G.; Li, H.; Michelagnoli, C.; Modamio, V.; Palacz, M.;
}

Title: $\quad$ Lifetime measurements with the Doppler Shift Attenuation Method using a thick homogeneous production target - Verification of the method

Year: $\quad 2017$

Version:

Please cite the original version:

Ertoprak, A., Cederwall, B., Jakobsson, U., Nyako, B. M., Nyberg, J., Davies, P., Doncel, M., De France, G., Kuti, I., Napoli, D. R., Wadsworth, R., Chugre, S.S., Raut, R., Akkus, B., Al-Azri, H., Algora, A., De Angelis, G., Ata, A., Bäck, T., . . Xiao, Z. G. (2017). Lifetime measurements with the Doppler Shift Attenuation Method using a thick homogeneous production target - Verification of the method. Acta Physica Polonica B, 48(3), 325-329. https://doi.org/10.5506/APhysPolB.48.325

All material supplied via JYX is protected by copyright and other intellectual property rights, and duplication or sale of all or part of any of the repository collections is not permitted, except that material may be duplicated by you for your research use or educational purposes in electronic or print form. You must obtain permission for any other use. Electronic or print copies may not be offered, whether for sale or otherwise to anyone who is not an authorised user. 


\section{LIFETIME MEASUREMENTS WITH THE DOPPLER SHIFT ATTENUATION METHOD USING A THICK HOMOGENEOUS PRODUCTION TARGET VERIFICATION OF THE METHOD*}

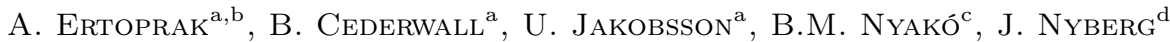
P. Davies ${ }^{\mathrm{e}}$, M. Doncel ${ }^{\mathrm{a}}$, G. De France ${ }^{\mathrm{f}}$, I. Kuti ${ }^{\mathrm{c}}$, D.R. Napoli ${ }^{\mathrm{g}}$

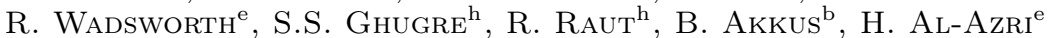
A. Algora ${ }^{i}$, G. De Angelis ${ }^{g}, A \cdot$ Atac $^{a}$, T. BäcK ${ }^{a}, A \cdot B$ Boso $^{j}$, E. Clément $^{f}$
D.M. Debenham ${ }^{\mathrm{e}}, Z_{\text {ss. Dombrádi }}^{\mathrm{c}}$, S. Erturk $^{\mathrm{k}}$, A. Gadea $^{\mathrm{i}}$, F. Ghazi Moradi ${ }^{\mathrm{a}}$

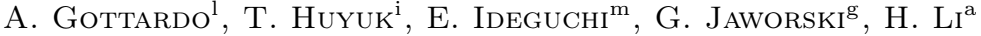
C. Michelagnoli ${ }^{\mathrm{f}}$, V. Modamio ${ }^{\mathrm{g}}$, M. Palacz ${ }^{\mathrm{n}}$, C.M. Petrache ${ }^{1}$, F. Rechia $^{\mathrm{j}}$

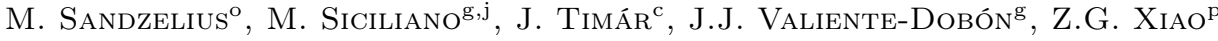

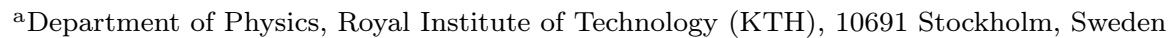

${ }^{\mathrm{b}}$ Department of Physics, Faculty of Science, Istanbul University

Vezneciler/Fatih, 34134 Istanbul, Turkey

${ }^{\mathrm{c}}$ MTA Atomki, 4001 Debrecen, Hungary

${ }^{\mathrm{d}}$ Department of Physics and Astronomy, Uppsala University, Uppsala, Sweden

e Department of Physics, University of York, Heslington, YO10 5DD York, UK

${ }^{\mathrm{f}}$ GANIL, CEA/DSM-CNRS/IN2P3, 14076 Caen Cedex 5, France

gINFN, Laboratori Nazionali di Legnaro, Legnaro, Italy

${ }^{\mathrm{h}}$ UGC-DAE CSR, Consortium for Scientific Research, Kolkata 700098, India

${ }^{\mathrm{i}}$ IFIC, CSIC-Universidad de Valencia, Valencia, Spain

${ }^{j}$ Dipartimento di Fisica e Astronomia, Universitá di Padova, Padova, Italy

${ }^{\mathrm{k}}$ Department of Physics, Faculty of Science and Arts

University of Ömer Halisdemir, 51240 Niğde, Turkey

${ }^{1}$ Centre de Sciences Nucléaires et Sciences de la Matière, CNRS/IN2P3, Université Paris-Saclay 91405 Orsay, France

${ }^{\mathrm{m}}$ Research Centre for Nuclear Physics, Osaka University, Osaka, Japan

${ }^{\mathrm{n}}$ Faculty of Physics, Warsaw University of Technology, 00-662 Warszawa, Poland

${ }^{\circ}$ University of Jyväskylä, Department of Physics, 40014 Jyväskylä, Finland

${ }^{\mathrm{P}}$ Department of Physics, Tsinghua University, Beijing 100084, China

\section{(Received March 6, 2017)}

Doppler Shift Attenuation Method (DSAM) analysis of excited-state lifetimes normally employs thin production targets mounted on a thick stopper foil ("backing") serving to slow down and stop the recoiling nuclei of interest in a well-defined manner. Use of a thick, homogeneous production target leads to a more complex analysis as it results in a substantial decrease in the energy of the incident projectile which traverses the target with an associated change in the production cross section of the residues as a function of penetration depth. Here, a DSAM lifetime analysis using

* Presented at the Zakopane Conference on Nuclear Physics "Extremes of the Nuclear Landscape", Zakopane, Poland, August 28-September 4, 2016. 
a thick homogeneous target has been verified using the Doppler broadened lineshapes of $\gamma$ rays following the decay of highly excited states in the semi-magic $(N=50)$ nucleus ${ }^{94} \mathrm{Ru}$. Lifetimes of excited states in the ${ }^{94} \mathrm{Ru}$ nucleus have been obtained using a modified version of the LINESHAPE package from the Doppler broadened lineshapes resulting from the emission of the $\gamma$ rays, while the residual nuclei were slowing down in the thick $\left(6 \mathrm{mg} / \mathrm{cm}^{2}\right)$ metallic ${ }^{58} \mathrm{Ni}$ target. The results have been validated by comparison with a previous measurement using a different (RDDS) technique.

DOI:10.5506/APhysPolB.48.325

\section{Experimental details}

The experiment was performed at the Grand Accélérateur National d'Ions Lourds (GANIL) in France. High-spin states in the semi-magic $(N=50)$ nucleus ${ }^{94} \mathrm{Ru}$ were populated in the ${ }^{40} \mathrm{Ca}\left({ }^{58} \mathrm{Ni}, 4 p\right){ }^{94} \mathrm{Ru}^{*}$ fusionevaporation reaction. The ${ }^{40} \mathrm{Ca}$ ions were accelerated to an energy of $150 \mathrm{MeV}$, degraded $128 \mathrm{MeV}$ in a thin Ta foil, and used to bombard target foils consisting of $99.9 \%$ isotopically enriched ${ }^{58} \mathrm{Ni}$ with the areal density of $6 \mathrm{mg} / \mathrm{cm}^{2}$, enough to stop the fusion products. The beam intensity varied between 5-10 $\mathrm{pnA}$ during 14 days of irradiation time. The $\gamma$ rays emitted from the reaction were measured using the EXOGAM germanium detector array [1] in its compact configuration comprising 11 large clover detectors. Each clover consists of four germanium crystals and each crystal is segmented in four quadrants of equal volume. Seven clover detectors were placed at $90^{\circ}$ and four detectors at $135^{\circ}$ with respect to the beam axis. The Ge detectors were surrounded by escape suppression shields consisting of BGO (bismuth germanate) scintillators to improve the spectrum quality. The fusion products corresponding to different reactions were selected using the DIAMANT $\mathrm{CsI}(\mathrm{Tl})$ charged-particle detector system [2, 3] which consisted of 80 CsI scintillators, and the Neutron Wall liquid scintillator detector array [4], consisting an array of 50 organic liquid scintillator detectors covering a $1 \pi$ solid angle in the forward direction. The trigger condition was fulfilled if one or more $\gamma$ rays was registered in the Ge detectors together with at least one neutron in the Neutron Wall. The latter signal was determined by a hardware threshold on the zero-crossing time of the neutron detector signals after passing through a shaping amplifier. The neutron hardware trigger was sufficiently relaxed to allow for a significant fraction of events which were not associated with neutron emission to be collected. In the off-line analysis, when needed, neutrons were selected cleanly by setting two-dimentional gates on the neutron time-of-flight versus the zero-crossover time. The energy calibration was performed using standard radioactive sources $\left({ }^{60} \mathrm{Co},{ }^{152} \mathrm{Eu}\right)$. 


\section{Data analysis and results}

The off-line analysis of selected $\gamma$-ray coincidence matrices and spectra was performed using the RADWARE software package [5]. The observation of Doppler broadened lineshapes also enabled the determination of level lifetimes using the Doppler Shift Attenuation Method (DSAM) [6]. In the standard DSAM measurements, a thin target coupled with a thick backing material ensures that the production cross section for the fusion-evaporation residues can be assumed to be constant across the target. Using a thick homogeneous production target results in a substantial decrease in the energy of the incident projectile as it traverses the target with an associated change in the production cross section of the residues as a function of penetration depth. Furthermore, only a certain fraction of the full target thickness will contribute significantly to the residue production in a given reaction channel, while the rest of the target thickness acts merely as the stopping medium, i.e. corresponds to the backing in a conventional measurement. The knowledge of the residue production rate as a function of target depth and the associated effective target thickness follows from the information on the production cross-section dependence on the beam energy and the evolution of the latter along the target thickness. The cross section for the production of the fusion residues as a function of beam energy can be obtained from experimental data and/or from statistical model calculations using, e.g., the PACE4 code [7]. Here, due to the strong variation of the fusion cross section close to the Coulomb barrier, the DSAM analysis is particularly challenging and we rely on experimental cross section data obtained by Bourgin et al. [8]. The stopping powers used in the analysis were calculated using the SRIM software package [9].

Lifetimes of excited states in the ${ }^{94} \mathrm{Ru}$ nucleus have been obtained from an analysis of the Doppler broadened lineshapes resulting from the emission of the $\gamma$ rays, while the residual nuclei were slowing down in the thick $\left(6 \mathrm{mg} / \mathrm{cm}^{2}\right)$ metallic ${ }^{58} \mathrm{Ni}$ target. The program LINESHAPE [10] in a modified version, see Ref. [11], was used to calculate the expected Doppler shape for a given $\gamma$-ray transition at a particular detector angle and perform leastsquare fits to the corresponding experimental spectrum in order to extract the level lifetime $(\tau)$. In this work, the method is verified using the $I^{\pi}=18^{+}$ state in the ${ }^{94} \mathrm{Ru}$ nucleus. The gamma-ray spectra have been generated with the gate on a transition below in the level scheme using (GTB) procedure [6]. The example of results of the fit of transitions lineshapes are shown in Fig. 1.

The results are given in Table I. The spin-parity assignments and transition energies proposed by Ghazi Moradi et al. [12] are adapted here. The data were sorted in $\left(E_{\gamma}-E_{\gamma}\right)$ matrices according to detector angle. The observed $\gamma$ rays of interest were analyzed for different angles simultaneously for the determination of the lifetimes. 

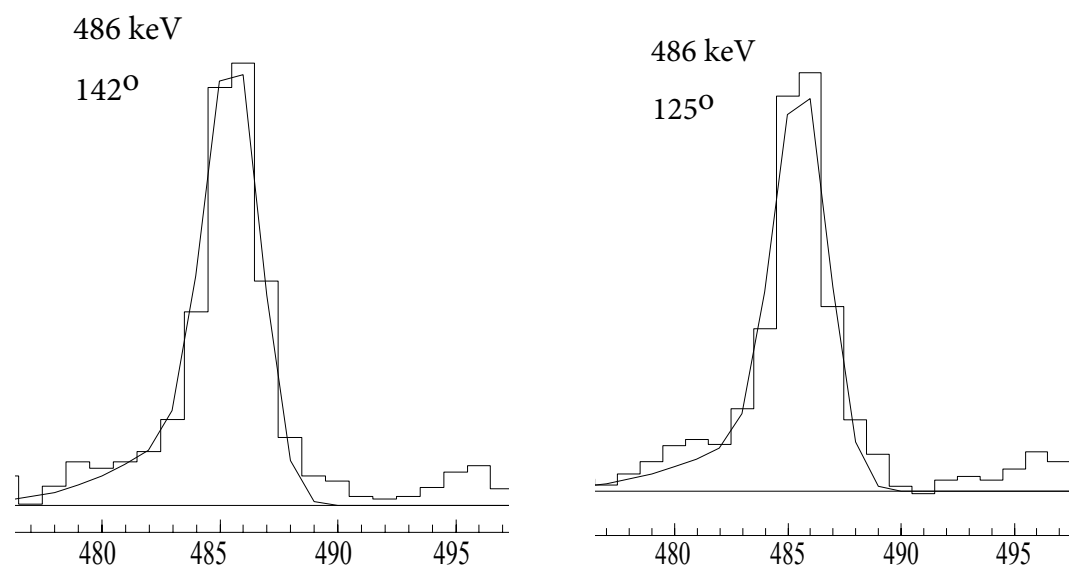

Fig. 1. Representative output fits of the Doppler shapes for the $486 \mathrm{keV}$ transition from the ${ }^{94} \mathrm{Ru}$ nucleus, obtained using the LINESHAPE package [11].

TABLE I

Lifetime value of the $18^{+}$level in the ${ }^{94} \mathrm{Ru}$ nucleus from the present work in comparison with the previously reported result, which employed the Recoil Distance Doppler Shift Method (RDDS).

\begin{tabular}{c|c|c|c}
\hline \hline$E_{\gamma}[\mathrm{keV}]$ & $I_{\mathrm{i}} \rightarrow I_{\mathrm{f}}$ & $\tau(\mathrm{ps})$ present work & $\tau(\mathrm{ps})$ literature [13] \\
\hline 486 & $18^{+} \rightarrow 17^{+}$ & $0.54(5)$ & $0.52(3)$
\end{tabular}

\section{Conclusions}

The lifetime of the $18^{+}$excited state in the ${ }^{94} \mathrm{Ru}$ nucleus has been measured and used to benchmark the applicability of the DSAM technique for a fusion-evaporation reaction at the Coulomb barrier with a thick homogeneous production target. The results agree with the lifetime measured previously using the RDDS technique, hence confirming the validity of the approach. In addition, lifetime values have been measured for the first time for the $19^{+} \rightarrow 18^{+}, 17^{+} \rightarrow 16^{+}$and $15^{+} \rightarrow 14^{+}$M1 transitions in the yrast cascade. These results will be reported elsewhere [14]. 
Lifetime Measurements with the Doppler Shift Attenuation Method Using ... 329

\section{REFERENCES}

[1] J. Simpson et al., Acta Phys. Hung. New Ser.: Heavy Ion Phys. 11, 159 (2000) http://pro.ganil-spiral2.eu/laboratory/detectors/exogam/exogam_aphns_11.pdf; http://pro.ganil-spiral2.eu/laboratory/detectors/exogam/exogam-detectors

[2] J.N. Scheurer et al., Nucl. Instrum. Methods Phys. Res. Sec. A 385, 501 (1997).

[3] J. Gál et al., Nucl. Instrum. Methods Phys. Res. Sec. A 516, 502 (2004).

[4] O. Skeppstedt et al., Nucl. Instrum. Methods Phys. Res. Sec. A 421, 531 (1999).

[5] D.C. Radford, Nucl. Instrum. Methods Phys. Res. Sec. A 361, 290 (1995).

[6] F. Brandolini, R.V. Ribas, Nucl. Instrum. Methods Phys. Res. Sec. A 417, 150 (1998).

[7] A. Gavron, Phys. Rev. C 21, 230 (1980).

[8] D. Bourgin et al., Phys. Rev. C 90, 044610 (2014).

[9] www.srim.org

[10] J.C. Wells, N.R. Johnson, ORNL Report 6689,44 (1991).

[11] R. Bhattacharjee et al., Phys. Rev. C 90, 044319 (2014).

[12] F. Ghazi Moradi et al., Phys. Rev. C 89, 014301 (2014).

[13] A. Jungclaus et al., Phys. Rev. C 60, 014309 (1999).

[14] A. Ertoprak et al., to be published. 